\title{
Stabilized vortex-antivortex molecules in a superconducting microdisk with a magnetic nanodot on top
}

\begin{abstract}
M. V. Milošević,* G. R. Berdiyorov, and F. M. Peeters ${ }^{\dagger}$
Departement Fysica, Universiteit Antwerpen, Groenenborgerlaan 171, B-2020 Antwerpen, Belgium

(Received 26 October 2006; revised manuscript received 20 December 2006; published 28 February 2007)

Symmetry-induced vortex-antivortex molecules in submicrometer superconducting polygons in homogeneous magnetic field became of general interest following the prediction of Chibotaru et al. [Nature (London) 408, 833 (2000)]. Recently, Carballeira et al. [Phys. Rev. Lett. 95, 237003 (2005)] found that these fascinating structures can be enforced by a magnetic dot placed on top of the sample. Here, we show that vortex-antivortex configurations can actually be induced in a superconducting disk by the above magnet with perpendicular magnetization, in spite of the nonzero net flux penetrating the sample and the absence of polygonal geometrical constraints. Our study is done within the Ginzburg-Landau formalism and shows that confinement makes vortex-antivortex states metastable (i.e., with higher energy) compared to the conventional vortex states; nevertheless, these states can be experimentally observed, and we propose a procedure for their realization, under a magnet with tilted magnetic moment.
\end{abstract}

DOI: 10.1103/PhysRevB.75.052502

PACS number(s): 74.78.Na, 74.20.De, 74.25.Dw

Mesoscopic superconductor-ferromagnet (SC-FM) hybrids became one of the prominent objects of study over the past decade, following the substantial progress in submicrometer lithography. ${ }^{1}$ Most of the work was inclined toward regular arrays of magnets on top of superconducting films, with emphasis on magnetic vortex pinning, and consequent technologically relevant enhancement of the critical current. ${ }^{2,3}$ Subsequent studies have also shown that the upper critical field of such SC-FM samples is significantly larger than that of conventional superconductors, ${ }^{4,5}$ due to compensation of the applied magnetic field and the stray field of the magnets. A careful investigation of the latter phenomenon has shown that magnets may induce vortex-antivortex structures in the underlying film, ${ }^{6,7}$ which then strongly interact with external flux lines, and exhibit distinct dynamical behavior. ${ }^{8}$

In parallel with the above studies, intense research was conducted on truly mesoscopic systems (in all three dimensions), i.e., superconducting disks and polygons with a magnetic dot on top. For example, transport measurements of Golubović et al. have revealed unique features, such as the controllable shift of the Little-Parks-like $H-T$ phase boundary ${ }^{9}$ and the expansion of the stability of particular vortex states. ${ }^{10}$ In Ref. 11, the appearance of the vortexantivortex configurations in the latter system was predicted theoretically, but only for zero total magnetic flux penetrating the SC. However, in reality, this condition cannot be satisfied for finite-size samples.

The question of vortex-antivortex structures in microsamples took a real upturn after Chibotaru et al. predicted their appearance even in a homogeneous magnetic field. ${ }^{12}$ While in the case of inhomogeneous field (such as one emerging from a magnet) vortex-antivortex states are products of the varying field polarity, in superconducting polygons, their appearance is imposed by the symmetry of the sample. ${ }^{13}$ Up to now, these vortex states have not been experimentally observed due to extreme proximity of vortices and antivortices, which makes them undistinguishable (unless they are conveniently pinned, see Ref. 14). Potential solution has been offered in Ref. 15, where vortices were spread further apart from the central antivortex by a weak magnet placed on top of the sample.

In the competition between the symmetry of a superconductor and the inhomogeneous stray field of the magnet, it is unclear which of them is most responsible for the vortexantivortex nucleation. To achieve a better understanding, we consider here a magnetic dot magnetized out of plane on top of a circular superconducting disk (separated by an insulating layer to avoid electronic coupling, see Fig. 1). We investigate the yet unexplored possibility of having stable vortexantivortex configurations in the finite sample, exposed to a nonzero total flux, and without any specific symmetry (e.g., triangle or square) that might impose vortex-antivortex states. The parameter range for their stability and their behavior in an external homogeneous magnetic field and for tilted magnetization of the magnetic dot are investigated in order to direct future experiments.

(a) Theoretical formalism. For thin superconductors (thickness $d<\xi, \lambda$ ), one may average the Ginzburg-Landau (GL) equations over the sample thickness and write them as

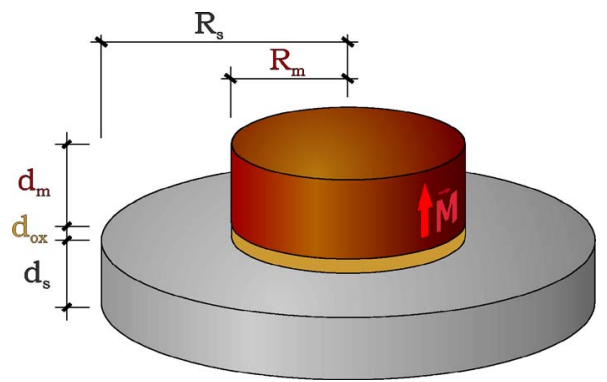

FIG. 1. (Color online) Oblique view of the sample-a superconducting disk (with radius $R_{s}$ and thickness $d_{s}$ ) under a magnetic dot (radius $R_{m}$, thickness $d_{m}$, and magnetization $\vec{M}$ ) and a separating insulating and/or oxide layer (thickness $d_{o x}$ ). 

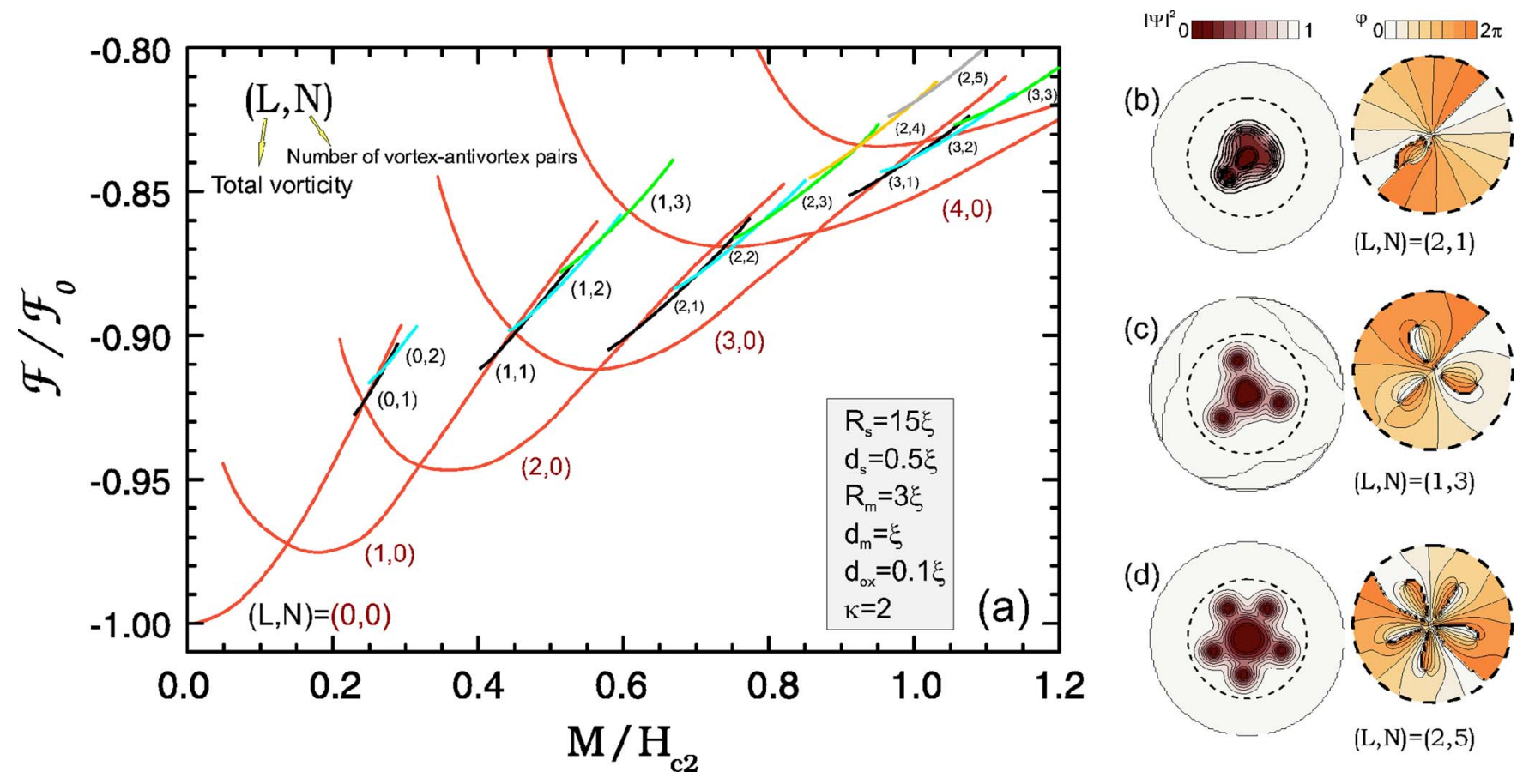

FIG. 2. (Color online) (a) The Gibbs free energy of the superconducting disk (in units of $\mathcal{F}_{0}=H_{c}^{2} / 8 \pi$ ) as a function of the magnetization of the magnetic dot placed on top. [(b)-(d)] Contour plots of the Cooper-pair density (left figures) and the distribution of the superconducting phase (zoomed) for three vortex-antivortex configurations.

$$
\begin{gathered}
\frac{\partial \Psi}{\partial t}=(-i \vec{\nabla}-\vec{A})^{2} \Psi-\Psi\left(1-|\Psi|^{2}\right), \\
-\Delta \vec{A}=\frac{d}{\kappa^{2}}\left[\frac{1}{2 i}\left(\Psi^{*} \vec{\nabla} \Psi-\Psi \vec{\nabla} \Psi^{*}\right)-|\Psi|^{2} \vec{A}\right] \delta(z),
\end{gathered}
$$

where the Laplacian still operates in all three dimensions, $\vec{A}$ is the total vector potential resulting from the magnetic dot and induced supercurrents, and $\kappa(=\lambda / \xi)$ denotes the GL parameter.

All distances are measured in units of the coherence length $\xi$, the vector potential in $c \hbar / 2 e \xi$, and the magnetic field in $H_{c 2}=c \hbar / 2 e \xi^{2}=\kappa \sqrt{2} H_{c}$. To solve the system of Eqs. (1) and (2), we apply a finite-difference representation of the order parameter and the vector potential on a uniform Cartesian space grid $(x, y)$, typically with 512 grid points in each direction, and use the link variable approach ${ }^{16}$ to discretize Eq. (1). Subsequent Gauss-Seidel iterative procedure ${ }^{17}$ allows us to find the stationary solution for $\Psi$. The vector potential is then obtained from Eq. (2) using fast Fourier transform, with boundary condition $A=A_{0}$ far away from the sample $\left(A_{0}\right.$ being the numerically calculated vector potential of the magnetic dot and/or homogeneous field). The dimensionless Gibbs free energy is calculated as $\mathcal{F}=V^{-1} \int[2(\vec{A}$ $\left.\left.-\vec{A}_{0}\right) \vec{j}-|\Psi|^{4}\right] d \vec{r} \vec{r}^{17}$ where $\vec{j}$ denotes the local supercurrent and $V$ is the sample volume.

To explore the superconducting state, we search for the self-consistent solution of Eqs. (1) and (2), coupled to the Neumann boundary condition $\left.(-i \nabla-\vec{A}) \psi\right|_{n}=0$. We gradually sweep up and/or down the magnetization of the magnetic dot
$M$ and recalculate each time the exact vortex structure and the corresponding Gibbs free energy. Our results are shown in Fig. 2 for a magnetic dot with radius $R_{m}=3.0 \xi$ and thickness $d_{m}=\xi$ on top of the superconducting disk with radius $R_{s}=15.0 \xi$ and thickness $d_{s}=0.5 \xi$; the thickness of the oxide layer $d_{o x}=0.1 \xi$ and $\kappa=2$, which approximately correspond to the experimental values found for $\mathrm{Nb}$ or $\mathrm{NbSe}_{2}$ samples. The above parameter values will be used throughout this Brief Report.

(b) Vortex-antivortex states. In our previous work, ${ }^{11}$ an expansion of the superconducting order parameter in the basis of eigenfunctions of the linearized GL equation was used, where we showed that a magnetic dot on top of the superconducting disk always induces vortex states with positive vorticity due to the positive total flux penetrating the sample. Although the stray field of the magnetic dot changes polarity in the vicinity of the perimeter of the dot, we did not find evidence of stable vortex-antivortex pairs. Quite contrary, vortex-antivortex states with zero total vorticity were imposed and easily stabilized in an infinite superconducting film, in the so-called Wigner-molecule configurations. ${ }^{6}$ Figure 2 shows the free energy and the possible vortex states for the intermediate case of a large but finite-size superconducting disk.

At first glance, red energy curves in Fig. 2(a) resemble the ones from Ref. 11 for the corresponding vorticity. Indeed, those vortex states are quite conventional, with giant vortex or a multivortex ${ }^{17,18}$ with vorticity $L$ nucleating under the magnetic dot. However, a crucial difference is observed close to the upper critical limit of each of these energy curves, where these states are found. Namely, the sharp gradient of the magnetic field under the dot edge may induce supercur- 


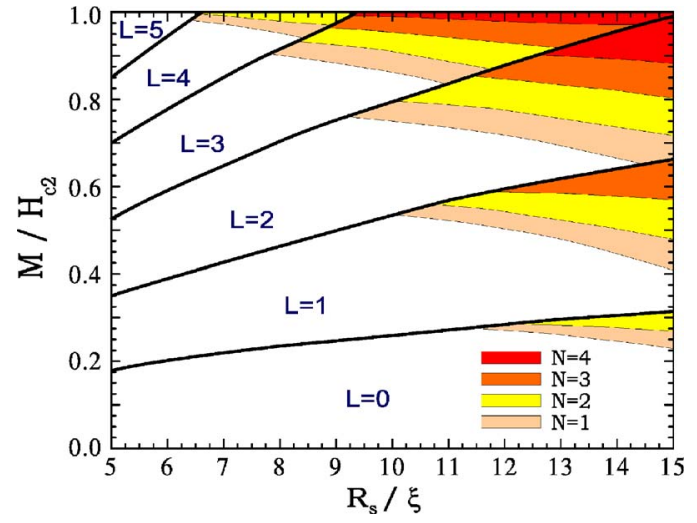

FIG. 3. (Color online) The phase diagram for stabilized vortexantivortex configurations. For each vorticity $L$ (separated by thick solid lines), colored regions give the range of parameters where states with $N$ vortex-antivortex pairs minimize the energy.

rents that exceed the depairing current with increasing magnetization of the dot. This triggers the vortex-antivortex nucleation, ${ }^{6}$ which remains stable in the absence of boundaries. In the present case, the sample boundaries are far from the nucleation point. In other words, the expulsion potential dip (opposite from the Bean-Livingston barrier) is located close to the sample edge and is, therefore, not affecting the antivortex, which is pinned near the center of the sample by its attraction to the vortex confined under the magnetic dot. Still, strictly speaking, the expulsion of the antivortex does minimize the energy, making states with vortex-antivortex pairs always metastable. Figures 2(b)-2(e) show the Cooperpair density and phase profiles ${ }^{19}$ of several vortex-antivortex states found (with total vorticity $L$ and $N$ vortex-antivortex pairs present).

Figure 3 shows the phase diagram of the lowest-energy

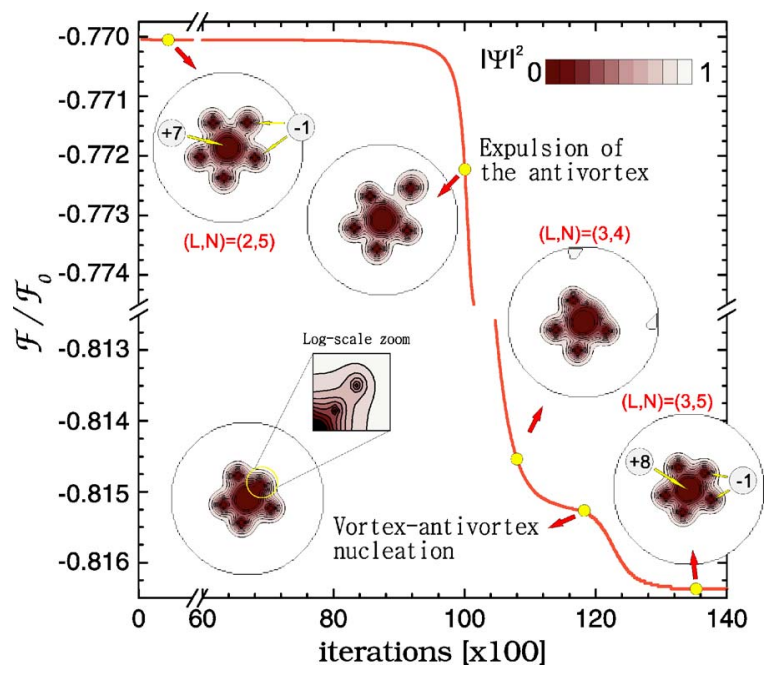

FIG. 4. (Color online) Behavior of the (2,5) vortex-antivortex state in a homogeneous magnetic field $\left(H=0.02 H_{c 2}\right.$, parallel to $\vec{M}$, $\left.M=1.05 H_{c 2}\right)$ : the Cooper-pair density plots illustrate the increase of total vorticity through expulsion of one antivortex [transition to $(3,4)$ state] and a subsequent nucleation of a another vortexantivortex pair [transition to a $(3,5)$ state].
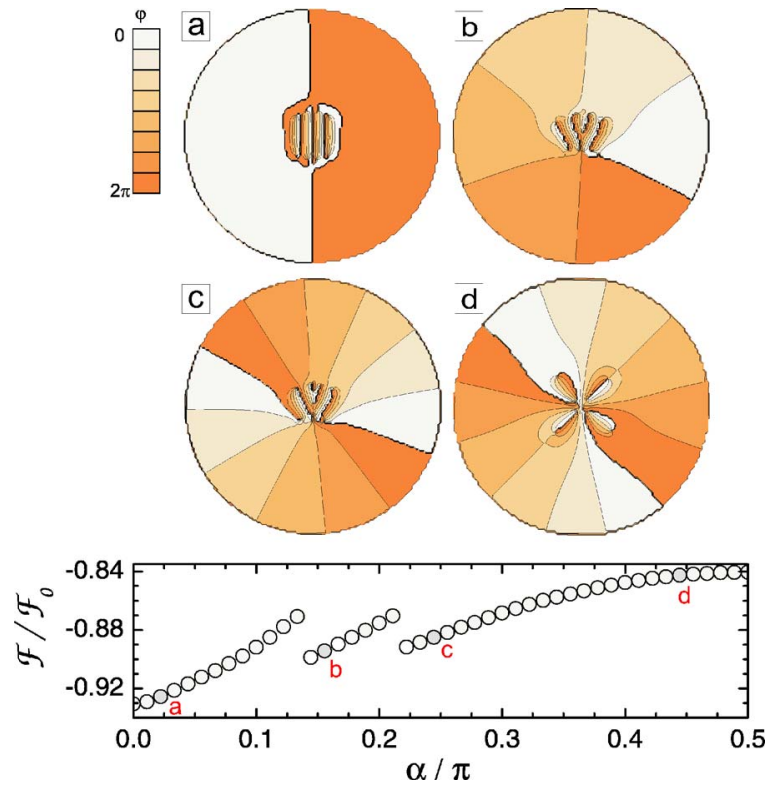

FIG. 5. (Color online) Evolution of the vortex-antivortex configurations during the gradual tilting of the magnetization of the magnetic dot (with angle $\alpha=0-\frac{\pi}{2}$ with respect to the sample plane, $\left.M=0.9 H_{c 2}\right)$. The contour plots $[(\mathrm{a})-(\mathrm{d})]$ depict the superconducting phase for the states found on this path [i.e., for $(L, N)=(0,4),(1,4)$, and $(2,4)$ twice, respectively].

vortex-antivortex configurations for a given vorticity as a function of the size of the superconducting disk and the magnetization of the dot. This diagram emphasizes two key features: (i) vortex-antivortex configurations become unstable for smaller $R_{s} / R_{m}$ ratio and the number of nucleated vortexantivortex pairs $N$ for given vorticity $L$ decreases and (ii) for fixed $R_{s} / R_{m}$ ratio, the maximal $N$ increases with increasing total vorticity. Feature (i) follows from the proximity of the sample boundaries, while feature (ii) can be understood from the enhanced stabilization of the antivortex shell by the enforced attraction to the larger vortex nucleus.

(c) Response to a homogeneous magnetic field. In what follows, we briefly discuss the influence of an additional magnetic field on the vortex-antivortex configurations. Namely, even a small external field is capable of inducing extra vortices in our sample due to the large size of the superconducting disk, and it is by no means trivial how the vorticity increases in the presence of vortex-antivortex pairs. To investigate this, we start the simulation from a state with vorticity $L=2$ and $N=5$ vortex-antivortex pairs found for $M=1.05 H_{c 2}$ (see Fig. 2) and apply a weak homogeneous field $H=0.02 H_{c 2}$ in the direction parallel to the magnetization of the magnetic dot. The subsequent time evolution of the superconducting state is shown in Fig. 4. Each iteration step in Fig. 4 denotes an interval approximately equal to the Ginzburg-Landau time $\left(\tau_{\mathrm{GL}}\right)$.

The insets of Fig. 4 show the snapshots of the Cooper-pair density profiles during the time evolution. Since the applied field results in an additional flux of $\Delta \Phi=2.25 \Phi_{0}$, the vorticity in the sample increases. However, instead of a vortex entry, we observed an antivortex exit. Consequently, one vortex-antivortex pair decouples, and the excess vortex re- 
mains in the sample [i.e., the $(2,5)$ configuration becomes a $(3,4)$ one]. The antivortex exit is caused by strong screening currents, which decrease the expulsion barrier at the sample edge. At the same time, supercurrents induced by an additional field superimpose on the existing currents throughout the sample. Therefore, the maximal current under the edge of the magnet (see Ref. 6) may be increased over the critical value, and another vortex-antivortex pair may nucleate. This indeed takes place in the present case, as shown in Fig. 4, and the superconducting state relaxes in a $(3,5)$ configuration. Note that the final vortex state depends on all relevant parameters and could be, in principle, any state with lower energy than the initial one.

(d) Experimental realization. As we have shown in Fig. 2, the predicted vortex-antivortex states are found only as metastable, i.e., with higher energy than the ground state. Therefore, they are arguably more difficult for experimental observation than conventional vortex states. At this point, we recall the properties of small superconductors with a centered in-plane magnet on top, ${ }^{20}$ where due to the zero total flux penetrating the sample only states with zero vorticity can exist. Since in this system vortex-antivortex states are easily stabilized, we employ this feature to facilitate their experimental observation in the present system. Namely, the magnetic dot can be initially prepared using a tilted magnetization with respect to the superconductor plane, where the tilting angle $\alpha$ favors vortex-antivortex nucleation. A small applied field can further straighten the magnetization and gradually stabilize vortex-antivortex states in a metastable regime. Figure 5 shows the free energy and the states found during the simulated tilting of the magnetization of the dot ( $M=0.9 H_{c 2}$ and other parameters same as in Fig. 2). As shown in four successive contour plots in Fig. 5, for $\alpha=0$, four vortices and four antivortices nucleate under the opposite poles of the magnet. When increasing $\alpha$, vortices stay confined under the dot while antivortices gradually move to the periphery and rearrange in a shell. During this process, the total flux penetrating the superconductor increases and, in our case, leads to the increase of vorticity through first-order transitions for $\alpha=0.12 \pi$ and $0.23 \pi$. Eventually, for $\alpha=\pi / 2$, we are left with a stable $(L, N)=(2,4)$ state, which in Fig. 2 had four other states with lower energy for $M=0.9 H_{c 2}$.

For direct observation of the vortex-antivortex states, besides the conventional vortex imaging techniques, the MTJ method can be considered, ${ }^{18}$ as well as transport measurements in a Corbino geometry, ${ }^{21}$ where vortices and antivortices should exhibit opposing dynamics.

This work was supported by the Flemish Science Foundation (FWO-Vl), the Belgian Science Policy (IUAP), the ESF-AQDJJ network, and the joint JSPS-ESF program Nanoscience and Engineering in Superconductivity.
*Present address: Department of Physics, University of Bath, Claverton Down, Bath BA2 7AY, United Kingdom.

†Electronic address: francois. peeters@ua.ac.be

${ }^{1}$ J. I. Martin, M. Vélez, J. Nogués, and I. K. Schuller, Phys. Rev. Lett. 79, 1929 (1997).

${ }^{2}$ J. I. Martin, M. Vélez, A. Hoffmann, I. K. Schuller, and J. L. Vicent, Phys. Rev. Lett. 83, 1022 (1999).

${ }^{3}$ M. J. Van Bael, K. Temst, V. V. Moshchalkov, and Y. Bruynseraede, Phys. Rev. B 59, 14674 (1999).

${ }^{4}$ M. Lange, M. J. Van Bael, Y. Bruynseraede, and V. V. Moshchalkov, Phys. Rev. Lett. 90, 197006 (2003).

${ }^{5}$ M. V. Milošević and F. M. Peeters, Europhys. Lett. 70, 670 (2005).

${ }^{6}$ M. V. Milošević and F. M. Peeters, Phys. Rev. B 68, 024509 (2003).

${ }^{7}$ M. V. Milošević and F. M. Peeters, Phys. Rev. Lett. 93, 267006 (2004)

${ }^{8}$ M. Lange, M. J. Van Bael, A. V. Silhanek, and V. V. Moshchalkov, Phys. Rev. B 72, 052507 (2005).

${ }^{9}$ D. S. Golubović, W. Pogosov, M. Morelle, and V. V. Moshchalkov, Appl. Phys. Lett. 83, 1593 (2003); Europhys. Lett. 65, 546 (2004).

${ }^{10}$ D. S. Golubović, M. V. Milošević, F. M. Peeters, and V. V. Moshchalkov, Phys. Rev. B 71, 180502(R) (2005).
${ }^{11}$ M. V. Milošević, S. V. Yampolskii, and F. M Peeters, Phys. Rev. B 66, 024515 (2002).

${ }^{12}$ L. F. Chibotaru, A. Ceulemans, V. Bruyndoncx, and V. V. Moshchalkov, Nature (London) 408, 833 (2000).

${ }^{13}$ For example, in a superconducting square for vorticity 3 , the state consisting of four vortices and a central antivortex may have lower energy than the configuration of three equidistant vortices, which break the square symmetry.

${ }^{14}$ R. Geurts, M. V. Milošević, and F. M. Peeters, Phys. Rev. Lett. 97, 137002 (2006).

${ }^{15}$ C. Carballeira, V. V. Moshchalkov, L. F. Chibotaru, and A. Ceulemans, Phys. Rev. Lett. 95, 237003 (2005).

${ }^{16}$ R. Kato, Y. Enomoto, and S. Maekawa, Phys. Rev. B 47, 8016 (1993).

${ }^{17}$ V. A. Schweigert, F. M. Peeters, and P. S. Deo, Phys. Rev. Lett. 81, 2783 (1998).

${ }^{18}$ A. Kanda, B. J. Baelus, F. M. Peeters, K. Kadowaki, and Y. Ootuka, Phys. Rev. Lett. 93, 257002 (2004).

${ }^{19}$ The encircling discontinuity of $2 \pi(-2 \pi)$ in the superconducting phase contour plot signals a vortex (antivortex).

${ }^{20}$ M. V. Milošević, G. R. Berdiyorov, and F. M. Peeters, Phys. Rev. Lett. 95, 147004 (2005).

${ }^{21}$ M.-C. Miguel and S. Zapperi, Nat. Mater. 2, 477 (2003); V. R. Misko and F. M. Peeters, Phys. Rev. B 74, 174507 (2006). 